\title{
The Quantum Measurement Problem and Physical reality: A Computation Theoretic Perspective*
}

\author{
R. Srikanth' \\ Poornaprajna Institute of Scientific Research, Devanahalli, Bangalore 562 110, India, and \\ Raman Research Institute, Sadashiva Nagar, Bangalore- 560 086娪
}

\begin{abstract}
Is the universe computable? If yes, is it computationally a polynomial place? In standard quantum mechanics, which permits infinite parallelism and the infinitely precise specification of states, a negative answer to both questions is not ruled out. On the other hand, empirical evidence suggests that NP-complete problems are intractable in the physical world. Likewise, computational problems known to be algorithmically uncomputable do not seem to be computable by any physical means. We suggest that this close correspondence between the efficiency and power of abstract algorithms on the one hand, and physical computers on the other, finds a natural explanation if the universe is assumed to be algorithmic; that is, that physical reality is the product of discrete subphysical information processing equivalent to the actions of a probabilistic Turing machine. This assumption can be reconciled with the observed exponentiality of quantum systems at microscopic scales, and the consequent possibility of implementing Shor's quantum polynomial time algorithm at that scale, provided the degree of superposition is intrinsically, finitely upper-bounded. If this bound is associated with the quantum-classical divide (the Heisenberg cut), a natural resolution to the quantum measurement problem arises. From this viewpoint, macroscopic classicality is an evidence that the universe is in $\mathbf{B P P}$, and both questions raised above receive affirmative answers. A recently proposed computational model of quantum measurement, which relates the Heisenberg cut to the discreteness of Hilbert space, is briefly discussed. A connection to quantum gravity is noted. Our results are compatible with the philosophy that mathematical truths are independent of the laws of physics.
\end{abstract}

PACS numbers: 03.65.Ta, 03.67.Mn

Keywords: quantum computation, complexity theory, computability, quantum measurement problem

\section{INTRODUCTION}

The advance of quantum information and quantum computation [1, 2] as a serious field of research has brought forth a new language for looking at problems in physics and a novel way of characterizing physical theories 3, 4]. Further, information processing may play a fundamental role in our understanding of physical laws [5, [6, 7, [] ]. Conversely, the discovery of Shor's algorithm [9] suggested that knowledge of the physical world is relevant to study the limits of efficient computation. These developments highlight the interplay between insights into information and computation developed by computer scientists on the one hand, and well-tested physical theories documented by physicists on the other. Although the idea that information theory or computer science can provide powerful ways to describe and explore the consequences of physical laws may be acknowledged as intuitively appealing, belief in its usefulness as a guide to new physics is unlikely to be widespread among physicists. We hope this work can help elucidate the connection between the two fields. Indeed, several independent approaches inspired by a similar philosophy have been undertaken 10, 11, 12, 13].

The eminent physicist J. Wheeler has suggested that phys-

\footnotetext{
* (C)2006 American Institute of Physics. The article appeared in Quantum Computing: Back Action 2006, IIT Kanpur, India, March 2006, Ed. Dr. D. Goswami, AIP Conference Proceedings 864, pp. 178-193 (2006).

$\dagger$ Electronic address: srik@rri.res.in

$¥$ Affiliation updated.
}

ical reality itself may be a manifestation of information processing through his idea expressed as the aphorism "it from bit" [14]. To quote Wheeler:
"It from bit" symbolizes the idea that every item of the physical world has at the bottom- at the very bottom, in most instances- an immaterial source and explanation; that which we call real- ity arises from the posing of yes-no questions, and the registering of equipment-invoked responses; in short, that things physical are information the- oretic in origin.

In this work, we suggest that the known power of physical computers and the quantum measurement problem together offer a clue to and suggest a particular realization of the above idea.

We believe that this approach highlights how computation theoretic considerations can shed light on fundamental questions about why physical laws are the way they are, and also help constrain or uncover new physics. For example, it is an interesting question how the theoretical model of computation compares with physical computers. For concreteness, one can ask whether NP-complete problems can be solved in polynomial time using the resources of the physical universe. What can we say about physics depending on whether the answer is affirmative or negative? These are some questions we consider here.

Doubtless, various profound approaches to such questions exist, among them string theory, different theories of quantum gravity, particle physics, etc. Various recondite clues, such as the black hole information paradox, dark matter, dark energy, gamma ray bursts are potential harbingers of new physics. Perhaps an advantage of the (quantum) information 
and computation theoretic approach such as this is that it may be possible to test predictions using relatively accessible optical and quantum optical experimental techniques.

The article is divided as follows. In the next section, we note that $\mathbf{N P}$-complete problems are found to be intractable in the physical world. This idea can be brought into perspective by showing that if quantum mechanics (QM) were not linear, or not unitary, or not local (ie., conforming to the nosignalling theorem), or not conforming to the Born $|\psi|^{2}$ rule [1], more efficient models of computation would be possible than believed to exist [15, 16]. In the subsequent section, we note that algorithmically uncomputable problems are believed to be unsolvable in the physical world. The question as to why Nature seems to be exactly as efficient and powerful as theoretical models of computation is considered thereafter. It is pointed out that one possible solution is that Nature is algorithmic: that is, physical reality is a manifestation of discrete sub-physical computations and information processing. We further indicate why this somewhat unusual interpretation receives clarification and support from the quantum measurement problem. The further Section recapitulates a computational model of quantum measurement, compatible with the conclusion of the preceding section. Possible implications for quantum gravity are then discussed before concluding in the last Section.

\section{COMPUTATIONAL COMPLEXITY CONSIDERATIONS}

In a remarkable work that founded computer science, in the course of studying the problem of what it means to be computable, Turing formalized the intuitive notion of an effective procedure or algorithm for a computational task by means of a class of abstract devices that have come to be called Turing machine (TM). A TM is a symbol-manipulating device, equipped with a movable tape of finite but unbounded length, divided into cells. Each cell is filled with an element from a finite alphabet which includes a blank symbol. A TM is equipped with a read-write head that possesses a property known as state, a finite set of instructions for how the head should modify the active cell, move the tape and alter its own state [17].

It is of interest to study the existence of efficient algorithms to perform certain tasks, the relevant resources being (memory) space and time (i.e., number of computational steps). The complexity class $\mathbf{P}$ denotes the set of all problems solvable on a TM in polynomial time, that is, in steps that are polynomial as a function of problem size. Problems in $\mathbf{P}$ are often considered as the class of computational problems which are "efficiently solvable" or "tractable". With the advent of probabilistic TMs [1] and then quantum TMs [18, 19], there are potentially larger classes, such as BPP (for 'bounded-error, probabilistic, polynomial time) and BQP (for 'bounded-error, quantum, polynomial time), that are considered tractable. A probabilistic TM can be considered as a (deterministic) TM with access to genuine randomness. BPP denotes the set of decision problems solvable on a probabilistic TM in polynomial time with error probability of at most $1 / 3$ on all instances. The choice of $1 / 3$, which is arbitrary, can be replaced with any constant between 0 and $1 / 2$. Analogously, BQP denotes the class of decision problems solvable by a quantum computer in polynomial time, with error prob- ability of at most $1 / 3[19]$. Obviously, $\mathbf{P} \subseteq \mathbf{B P P} \subseteq \mathbf{B Q P}$.

Roughly speaking, the set of decision problems whose solutions can be easily verified is called NP. More precisely, NP is the class of decision problems such that (a) to every positive instance of answer, there exists a polynomial-size proof verifiable by a deterministic polynomial-time algorithm (i.e., an efficiently computable witness of membership); and (b) to every negative instance of answer, the algorithm must declare invalid any purported proof that the answer is "yes" [20]. An example of a problem in NP is graph isomorphism (GI), the problem of determining whether two graphs on the same vertex set are isomorphic; here the witness is a permutation of the vertices that makes the two graphs equal.

A problem $P$ is in complexity class co-NP if and only if its complement $\bar{P}$ is in NP. In simple terms, co-NP is the class of problems for which efficiently verifiable proofs of "no"-instances, also called counterexamples, exist. The complement of GI, graph non-isomorphism, is clearly an example of a problem in co-NP. GI is not known to be in co-NP.

In simple terms, the class of NP-complete problems is the class of hardest problems in NP in the sense that if one can find a way to solve a NP-complete problem 'easily' (i.e., in polynomial time), then one can use that algorithm to solve all NP problems easily 21]. The Boolean satisfiability problem (SAT) is NP-complete (Given a boolean expression, is there at least one assignment of true/false values to the variables that makes the expression have a "true" value?). Closely related to the SAT problem is the TAUT problem, the problem of determining whether a given boolean formula is a tautology, which is co-NP-complete (Given a boolean expression, does every possible assignment of true/false values to the variables yields a true statement?). BQP is suspected, but not known, to be disjoint from the class of NP-complete problems and a strict superset of $\mathbf{P}$. Integer factorization and discrete log, which are in $\mathbf{B Q P}$, are NP problems suspected to be outside of both the class $\mathbf{P}$ and and also of the class of NPcomplete problems. Clearly, $\mathbf{P} \subseteq \mathbf{N P}$ since easy solvability implies easy verifiability. Intuitively, one would expect that the converse is not true. Yet, interestingly, this has in fact not been proved so far. This encapsulates the $\mathbf{P} \stackrel{?}{=} \mathbf{N P}$ problem, the most famous open question in computer science.

Computational complexity class \#P is the set of counting problems associated with the decision problems in NP. That is, it is the class of function problems of the form "compute $f(x)$ ", where $f$ is the number of accepting paths of an NP machine. Unlike most well-known complexity classes, it is not a class of decision problems, but a class of function problems. The canonical \#P problem is \#SAT: given a Boolean formula, compute how many satisfying assignments it has [20].

PSPACE is the set of decision problems solvable on a TM using polynomial amount of memory. It is immediate that $\mathbf{P} \subseteq$ PSPACE, since a poly-time algorithm can consume only polynomial space. Given a boolean formula using only $\exists$ (existential) quantifiers to bind the variables yields a problem in SAT; using only $\forall$ (universal) quantifiers yields a problem in TAUT. Alternating both yields a True Quantified Boolean formula (TQBF) problem, which is PSPACEcomplete. A decision problem is in PSPACE-complete if it is in PSPACE, and every problem in PSPACE can be reduced to it in polynomial time. For example, a SAT problem is the question of whether the following is true: $\quad \exists_{x_{1}} \exists_{x_{2}} \exists_{x_{3}} \exists_{x_{4}}\left(x_{1} \vee \neg x_{3} \vee x_{4}\right) \wedge\left(\neg x_{2} \vee x_{3} \vee \neg x_{4}\right)$. This can be generalized to a TQBF by replac- 
ing the above quantified Boolean formula by $\exists_{x_{1}} \forall_{x_{2}} \exists_{x_{3}} \forall_{x_{4}}\left(x_{1} \vee \neg x_{3} \vee x_{4}\right) \wedge\left(\neg x_{2} \vee x_{3} \vee \neg x_{4}\right)$. It is known that NP $\subseteq$ PSPACE. The following containments are known to hold: $\mathbf{P} \subseteq \mathbf{B P P} \subseteq \mathbf{B Q P} \subseteq \mathbf{P P} \subseteq \mathbf{P S P A C E}$ and $\mathbf{P} \subseteq \mathbf{N P} \subseteq \mathbf{P P} \subseteq \mathbf{P} \mathbf{P P A C E}[22]$.

In the remaining part of this Section, we consider variants of QM that lead to more powerful (in the sense of complexity) models of computation. QM is known to be an 'island in theoryspace': it is strictly linear, unitary and having measurements obey the Born $|\psi|^{2}$ rule [15]. One cannot give up even one of these features, without collapsing its whole structure, as viewed from some physical or computational perspective. In this sense, it is unlike, for example, gravity, where one can define a family of Brans-Dicke theories in the neighborhood of General Relativity that are practically indistinguishable from the latter.

Consider solving SAT on a computer powered by nonlinear QM. This is easily solved if we have a polynomial time algorithm that determines whether there exists an input value $x$ for which $f(x)=1$, where $f$ is a boolean black box function. To begin with, we assume $f(x)=1$ on at most one value of $x$. Prepare the state $|\psi\rangle=2^{-n / 2} \sum_{x=0}^{2^{n}-1}|x\rangle_{\text {index }}|f(x)\rangle_{\text {flag }}$ on $n$ qubits and a 'flag' qubit.

There are $2^{n-1} 4$-dim subspaces, consisting of the first index qubit and the flag qubit, labelled by the index qubits $2, \cdots, n$. On each such subspace, the first index qubit and flag qubit are in one of the states $|00\rangle+|11\rangle,|01\rangle+|10\rangle$, $|00\rangle+|10\rangle$. A 'nonlinear OR' is applied to these two qubits to transform them according to:

$$
\left.\left.\begin{array}{l}
|00\rangle+|11\rangle \\
|01\rangle+|10\rangle \\
|01\rangle+|11\rangle
\end{array}\right\} \longrightarrow|01\rangle+|11\rangle ; \quad|00\rangle+|10\rangle \longrightarrow|00\rangle+|10\rangle\right) .
$$

This operation is repeated $(n-1)$ times, pairing each other index qubit with the flag. The number of terms with 1 on the flag bit doubles with each operation so that after the $n$ operations, it becomes disentangled and can then be read off to obtain the answer [23]. A slight modification of this algorithm solves problems in \#P efficiently. One replaces the flag qubit with $\log _{2} n$ qubits and the 1-bit nonlinear OR with the corresponding nonlinear counting. The final read-out is then the number of solutions to $f(x)=1$.

One can also solve SAT efficiently via non-unitary QM [16]. For example, to the second register of $|\psi\rangle=$ $2^{-n / 2} \sum_{x}|x\rangle|f(x)\rangle$, apply the nonunitary but invertible gate

$$
G=\left(\begin{array}{ll}
2^{-2 n} & 0 \\
0 & 1
\end{array}\right)
$$

Measurement on the second register allows one to know whether there exists $x$ such that $f(x)=1$ with exponentially small uncertainty. This also solves the complement of SAT, to which TAUT is (polynomial-time many-one) reducible.

As another variant of QM, suppose QM allows the probability of measurement outcomes to depend on other norms $p$ than the 2-norm of Born's $|\psi|^{2}$ rule. Restricting the dynamics to be norm-preserving leaves only the trivial dynamics of generalized permutation matrices. So the only option seems to be to use manual normalization: to stipulate that when a state $|\psi\rangle=\sum_{x} \alpha_{x}|x\rangle$ is measured in the computational basis, the probability of outcome $x$ is $\left|\alpha_{x}\right|^{p} / \sum_{y}\left|\alpha_{y}\right|^{p}$. Since here norm is not required to be preserved, the dynamics is free to be unitary or simply consist of invertible matrices. In the latter case, 'local normalization' can be an option. Each of these three options can be shown to allow quantum computers to solve even PP-complete problems [22] in polynomial time [16], which are believed to be harder than NP-complete problems.

Similarly, allowing for nonlocal signalling also permits efficient solving of SAT. To see this, observe that nonlocal signalling almost always implies a departure from standard QM: nonlinearity (some instances include those discussed in Refs. [25, 26]), non-unitarity, etc. As an example of the latter case, we note that the gate in Eq. (2) can be used to transmit a nonlocal signal. To do so, sender Alice applies either $G$ or $X G X$ to her qubit in the entangled state $(1 / \sqrt{2})(|01\rangle+|10\rangle)$, shared between her and Bob. Accordingly, Bob finds $|0\rangle$ or $|1\rangle$ with probability exponentially close to 1 . Here $X$ is Pauli $X$ operator. This nonlocal signal 'instantaneously' transmits classical information without requiring material or energy transfer using only the Einstein-Podolsky-Rosen channel [24]. It is not surprising that nonlocal signalling power is closely related to the power to solve hard problems efficiently, inasmuch as a similar "communication across superposition branches" is required in both cases.

In fact nonlinear quantum computers can even solve PSPACE problems efficiently [16]. To solve the PSPACEcomplete problem mentioned above, one alternatively applies nonlinear OR's and AND's, (instead of only nonlinear OR's as used to solve SAT) to: $|\psi\rangle=\sum_{x=0}^{2^{n}-1}|x\rangle_{\text {index }}|f(x)\rangle_{\text {flag }}$, starting with $n$th and flag qubits, moving the control bit sequentially leftward. Here the one-bit nonlinear AND is analogous to Eq. (1), given by:

$$
\left.\left.\begin{array}{l}
|00\rangle+|10\rangle \\
|00\rangle+|11\rangle \\
|01\rangle+|10\rangle
\end{array}\right\} \longrightarrow|00\rangle+|10\rangle ; \quad|01\rangle+|11\rangle \longrightarrow|01\rangle+|11\rangle\right) .
$$

This will disentangle the flagbit which is then read off to obtain the answer.

The above observations raise the question as to why QM 'chooses' to be such an island in theoryspace- strictly linear, unitary, local and conforming to the Born $|\psi|^{2}$ rule. A similar observation applies to other promising candidates among natural processes that potentially offer more efficient models of computation, such as simulated annealing, soap bubbles, protein folding and 'relativistic computation' [16]. On closer inspection, the evidence in support of their ability to efficiently solve hard problems is not found to be persuasive. Their route to efficiency seems always to be blocked by such features as taking longer relaxation or evolution times [27], ending up in local minima, requiring exponentially large energy, etc.

These considerations lend support to the NP-hardness assumption (NPHA): that NP-complete problems are intractable in the physical world [16]. Our confidence in the probable veracity of this assumption stems not only from empirical knowledge of the physical world, but from noting that simple modifications to the laws of (quantum) physics, which could have led to the possibility of more effecient computing machines, are not found to be availed of in nature.

\section{COMPUTATABILITY CONSIDERATIONS}

Related to the issue of computational complexity is the question of computability, that is, the existence of an algo- 
rithm to solve a given computational task. An existential proof for uncomputable functions is based on a counting argument: the number of functions $f: \mathbf{N} \mapsto\{0,1\}$ is uncountably many $\left(2^{\aleph_{0}}\right)$, whereas the number of TMs is only countably infinite. Thus, most functions are (Turing) uncomputable. A specific example is Turing's halting problem, which is undecidable.

Suppose all TMs are uniquely numbered as $M_{j}(\cdot)(j=$ $0,1,2 \cdots)$ according to some consistent scheme. Consider the halting set $H \equiv\left\{j \mid M_{j}(j) \downarrow\right\}$, consisting of machine numbers of TMs that halt when they get as input their own number. Simply running $M_{j}(j)$ until it halts constitutes an algorithm to accept $H$, that is, to determine any "yes" instance to the problem of whether $j \in H$. Thus $H$ is semi-decidable or recursively enumerable (r.e.). The latter name derives from the fact that there is an enumeration procedure (employing a 'dovetailing' principle) whereby every element in $H$ is eventually detected.

But $H$ is not co-r.e. (i.e., its complement $\bar{H}$ is not r.e) because there is in general no algorithm to check the "no" instance of this question. Intuitively, this is because if a program does not halt, we would never know that it won't do so at a later time. More rigorously, suppose $\bar{H}$ is r.e: let $d$ be the machine or program number of the TM that accepts $\bar{H}$. Thus $n \notin H \Longleftrightarrow M_{d}(n) \downarrow$. Therefore: $d \notin H \Longleftrightarrow M_{d}(d) \downarrow$. However the definition of $H$ tells us that: $d \in H \Longleftrightarrow M_{d}(d) \downarrow$. Thus we have that: $d \in H \Longleftrightarrow d \notin H$, a contradiction. It follows that $\bar{H}$ is not r.e. $H$ is thus non-recursive- there exists no general membership algorithm for $H$. The halting function $h(x) \equiv\{x \mid x \in H\}$ is thus algorithmically uncomputable. Uncomputability implies [28] Gödel incompleteness [29].

One might ask whether uncomputability is a limitation of the TM model of computation, and whether perhaps an algorithm may be more general than a TM. According to the Church-Turing thesis (CTT), the answer is in the negative. CTT asserts that any problem that may be intuitively considered as computable (in a reasonable sense) is computable on a TM. That is, the formal concept of a TM captures exactly the intuitive idea of an algorithm or an effective procedure. Note that it is not provable, since it relates an intuition to a formal notion. Nevertheless, CTT is falsifiable in the sense that it can be refuted by the discovery of an intuitively acceptable algorithm or, more starkly, of some physical process, for a Turing-uncomputable task.

QM is characterized by infinite parallelism and the infinite precision of amplitudes (the continuum nature of Hilbert space) [30]. By the counting argument, the cardinality of the set of quantum TMs (or programs) equals that of all functions $f: \mathbf{N} \mapsto\{0,1\}$. Thus, the counting argument cannot be used to exclude a quantum algorithm from computing the halting function $h(x)$. In particular, one can conceive of a quantum machine $\mathcal{Q}$ that accepts $\bar{H}: n \notin H \Longleftrightarrow \mathcal{Q}(n) \downarrow$. Contradiction through self-reference is averted because $\mathcal{Q}$, being represented by a real number, cannot be the argument to any TM. (Actually, this argument can be applied also to realvalued TMs.)

Yet, it is usually believed that quantum Turing machines (QTM) can only compute the same functions that are computable with classical Turing machines. The QTM model, defined by Deutsch [18] and further formalized by Bernstein and Vazirani [19], is simulable by classical Turing machines (albeit at the expense of exponential slowdown), therefore so far as computability is concerned and within the scope of this QTM model, the set of computable functions remains the same as that for (classical) TM. Empirical evidence suggests that in computers based on the principle of relaxing to an energy minimum that encodes the solution, as DNA computer, soap bubbles, simulated annealing, etc., the physical relaxation time, which is a measure of computational complexity, tends to diverge as a function of problem size. Another possible impediment to super-Turing power is noise, which can render unfeasible infinitely precise computation.

We remark on a further point: that, even with infinite parallelism, quantum computers may need nonlinearity to solve non-recursive problems. This is analogous to the difficulty of simulating nondeterminism using the exponential parallelism of QTMs [31]. To see this, we briefly describe a model of infinite quantum parallelism.

We are given a problem $L \subseteq \mathbf{N}$ and an infinite dimensional quantum system, whose Hilbert space $\mathcal{H}_{S}$ is spanned by vectors $\{|j\rangle\}$. Suppose we can engineer a unitary transformation $U$ whose action on $\mathcal{H}_{S} \otimes \mathcal{H}_{R}$, where $\mathcal{H}_{R}$ is the 2-dimensional space of an ancilla, is such that $U|j\rangle|0\rangle=|j\rangle|f(j)\rangle$, where $f(j)=1$ if $j \in L$ and $f(j)=0$ otherwise. We assume further that we can arrange energetically so that all superpositional pathways $j$ terminate within finitely bounded time $T$ independent of $j$ (this requirement is analogous to invoking the linear speed-up lemma [32] in the model of infinite parallelism proposed in Ref. [33]).

Consider trying to solve the halting problem, or, equivalently, Hilbert's 10th problem 34]. In the former case, the action of $U$ on pathway $j$ may simulate a given program $P$ acting on its input $x$, through the first $j$ steps, and evaluate $f(j)$ to " 1 " iff $P(x)$ halts within these steps. In the latter problem, the quantum computer computes on each pathway $j$, the given Diophantine equation $D$ on input $j$ and evaluates $f(x)$ to " 1 " iff $D(j)=0$.

If $U$ were physically realizable, running this quantum computer on a superposition of all possible inputs, by virtue of quantum mechanical linearity, one obtains the possibly entangled state $U\left(\sum_{j=0}^{\infty}|j\rangle|0\rangle\right) \longrightarrow \sum_{j=0}^{\infty}|j\rangle|f(j)\rangle$. To be able to compute Turing-uncomputable functions, we require a finite method to detect an acceptance on at least one term in the output superposition of $U$. Unfortunately, we can do no better than to quantum search through all the infinite terms. We thus recover uncomputability, since a quantum search can yield no better than a quadratic speedup 31], so that a negative answer to $L$ can never be ruled out in this way in finite time. This line of reasoning suggests that infinite quantum parallelism cannot necessarily be harnessed to solve Turing uncomputable problems.

\section{THE QUANTUM MEASUREMENT PROBLEM}

The conclusions of the preceding two sections demonstrate that CTT and NPHA present us with what is arguably a twofold coincidence: (a) On the one hand, we find that NPcomplete problems do not seem to be efficiently solvable by any physical means; (b) on the other hand, algorithmically uncomputable problems do not seem to be computable by any physical means.

This $\underline{\text { close }}$ correspondence between the efficiency and power of theoretical algorithms and those of physical computers (abbreviated to CCAP) evokes the question: How do we account it? Three broad possibilities present themselves: 
(1) that the laws of physics support super-Turing efficiency and power, but instances of violation of NPHA and CTT in Nature remain unidentified.

(2) The universe is not Turing computable, let alone polynomial, but super-Turing efficiency or power is not "harnessable", because of quantum mechanical 'insularity' (i.e., QM being linear, unitary, etc.), or "accidents" such noise, energetics, etc. One possibility that realizes this option is that the universe is a quantum computer, where the origin of uncomputabality and intractability could be the infinite quantum parallelism (eg., as in a harmonic oscillator) and exponential quantum parallelism (eg., as in a multi-qubit system), respectively, with the purported impossibility of solving the halting problem or efficiently simulating nondeterminism having its origin in the special, 'insular' structure of QM.

(3) The universe is algorithmic: the states and evolution of physical objects are manifestations of discrete information stored and computations performed at the 'subphysical' level by a probabilistic TM, which serves as a 'meta-universe'. Physical laws are manifestations of efficient sub-physical algorithms on the probabilistic TM. Physical reality is fundamentally information theoretic, in the sense of Wheeler's phrase "it from bit". We need to clarify, which we do below, why this option does not contradict the fact that $\mathbf{B Q P}$ apparently characterizes the observed power of quantum computers.

Are there other observations that can influence our choice of one or other option? We claim that the quantum measurement problem (QMP) is one such. We will argue that QMP clarifies the above situation in two ways: first, it will enable us to argue that the apparent BQPness of microscopic quantum states that we are sure we can prepare does not contradict the proposition that classical macrosystems exist in BPP. This is crucial to justify option (3) as an explanation of CCAP; second, it will enable us to argue that macro-classicality undermines both options (1) and (2).

QMP is a fundamental problem of interpretting QM, that, in its simplest form, is concerned with the questions (among others): Why is the macro-world classical? Why do we not find conspicuous macroscopic manifestations of quantum interference?

In slightly more detail: when a quantum measurement is performed on a system $S$ in the state $|\psi\rangle=\sum_{j} \alpha_{j}|j\rangle$ $\left(\sum_{j}\left|\alpha_{j}\right|^{2}=1\right)$, quantum mechanical linearity implies that a Schrödinger cat state should be generated as follows:

$$
\sum_{j} \alpha_{j}|j\rangle|R\rangle \longrightarrow \sum_{j} \alpha_{j}|j\rangle\left|R_{j}\right\rangle
$$

where $|R\rangle$ is the 'ready' state, and $\left|R_{j}\right\rangle$ 's the correlated states of the measuring apparatus. Instead, selectively speaking (i.e., conditioned on the read-out of the measuring apparatus), only one of the possible outcomes $|j\rangle\left|R_{j}\right\rangle$ is observed. The origin of this apparently discontinuous, non-unitary jump, sometimes called the "collapse of the wavefunction" or "reduction of the state vector", is QMP (or, more precisely, an important part of QMP).

QMP is a long-standing and contentious problem about the interpretation of QM, with many proposed, interesting solutions (cf. 35] for a detailed review). Often the formal collapse is treated only as an interpretational concept rather than an objective physical phenomenon. Part of the reason is that it is hard to establish a critical size at which the physical collapse may be said to happen or to come up with a clearly testable mechanism. In the view of many, it is not ruled out that QMP may be resolved without invoking collapse. Still, in Refs. 36, 37], we argued that the mechanism of environmental decoherence [38, 39, 40], complemented by a wavefunction collapse, is a reasonable way to resolve QMP. Our support for option (3) over options (1) and (2) is based on this line of thought, in particular, the assumption that collapse is an objective occurance.

Under option (3), one is required to reconcile the assumed polynomiality (i.e, BPPness) of the universe with the observed exponentiality of the quantum states, which empowers the massive quantum parallelism behind Shor's algorithm [9]. The latter gives a polynomial time quantum algorithm for the factoring problem, which is not believed to be in $\mathbf{P}$. A crucial fact here is that this exponentiality has never been seen on macro-scales, which of course is QMP. Therefore, if we accept option (3), the only way to avoid the contradiction between the presumed polynomiality of the universe and the apparent exponentiality confirmed at small scales seems to be to fundamentally upper-bound the degree of superposition allowed for any quantum system. By choosing this bound judiciously, that is, by positioning it at the Heisenberg cut (the scale that seemingly separates the quantum realm from the classical), one can guarantee that sufficiently small systems will manifest superpositions and hence exponential behaviour; on the other hand, for sufficiently large systems, exponential (superpositional) behaviour will be replaced by polynomial (classical) behaviour. With this proviso, the fact that quantum computers are apparently characterized by BQP at microscopic scales would not be incompatible with the proposition that the universe is in BPP.

Conversely, this may be expressed as follows. We treat QMP interpreted as an objective collapse as a threshold phenomenon, which separates the quantum behaviour in the micro-world from the classical behaviour in the macro-world. It may be thought of as a sort of quantitative (as against qualitative) "Sure/Shor" separator [41], a criterion that separates the quantum states are that are surely experimentally preparable, from states that arise in a large-scale implementation of Shor's algorithm. Given the polynomiality of the universe per option (3), and the tested exponentiality at small scales, option (3) predicts that the quantum superposition principle should break down at some finite scale. By identifying this threshold scale with the quantum-classical divide, we have a natural resolution to QMP. From this viewpoint, macro-classicality is a sign that the universe is in BPP.

QMP interpreted as a collapse phenomenon in fact undermines both options (1) and (2). For example, consider the assumption of linearity of QM under option (2) as a possible explanation for CCAP. Clearly, the validity of the linearity of quantum evolution at all scales is called into question if lack of superpositions of the type (4) is due to collapse. This contradiction is not weakened by taking into consideration noise, measuring errors and such details [43]. (A caveat is that alternative interpretations of QM like Many-worlds or Bohmian may be exempt from this contradiction.) If we regard macroclassicality as a sign of breakdown of linearity at some scale, then option (2) seems to be disfavored.

This breakdown would also imply that a superposition of infinite terms is disallowed. In the ITS model, infinite parallelism is necessary (though not sufficient) for computing nonrecursive functions. According to this model, the breakdown 
in linearity/superposition principle also disfavors option (1).

Adopting option (3) as the explanation of CCAP, we are led to the worldview that the universe is computable, 'simulated/computed' by efficient algorithms run on a sub-physical probabilistic TM, which serves as a meta-universe. Quantum randomness is accepted as fundamental. Thus the universe is described as a polynomial place in BPP. We believe that option (3) is potentially a more 'natural' and 'deeper' explanation of CCAP than the other options. First, we note that NPHA and CTT follow immediately and naturally: CTT is simply the consequence of Turing-uncomputability; NPHA follows from the situation that probably no efficient algorithms exist to solve hard problems (if such exist, perhaps they are so unobvious that Nature hasn't yet 'discovered' them!) Under option (3), we then conclude that Schrödinger evolution is linear, unitary and compatible with no-signalling and quantum measurement obeys the Born rule because if it were not so, the resulting computational power would be larger than that supportable by an algorithmic universe. In fact, wavefunction collapse implies a breakdown in both linearity and unitarity, but not of a kind that would allow efficient computation of hard problems, or, for the matter, nonlocal signaling. (though non-selectively, that is, at the level of the density matrix, the evolution is still linear, and can be regarded as unitary in a larger Hilbert space [1].) This suggests that while the insular structure of QM in theory space is special, a departure of QM from insularity may be allowed if it is compatible with the algorithmicity of the universe.

These observations allow us to reduce a host of physical laws to basic results in computation theory. Furthermore, teleologically speaking, there is an obvious, intrinsic 'motivation' for an algorithmic universe to employ efficient algorithms: if they indeed suffice to engender a sufficiently complex universe (which seems to be the case, cf. Ref. [12]), then no further 'computational effort' on the universe's part is needed!

Options (1) and (2) are less satisfactory as fundamental explanations of CCAP. For example, consider the assumption of linearity of QM under option (2) as a possible explanation. There is no a priori reason to expect that a self-consistent QM should be linear. Invoking the prohibition on nonlocal signaling to impose linearity brings in an extraneous physical criterion. Moreover, it means that one of them has to accepted axiomatically.

From the viewpoint of classical computation, option (3) is conservative because it sides with the belief that it is unlikely that Nature would manipulate or maintain exponentially (not to mention, infinitely) large objects "free of cost" (or, at unit cost) 42]. It is intuitively satisfying to picture the laws of physics as algorithms for physical dynamics, and hence that the limits on efficient computation and on computability in the physical world derived from them, to correspond to formal, purely mathematical notions of computation. Option (3) thus arguably supports the philosophy that mathematical truths do not depend on the laws of physics and suggests that insights from computer science can be used to constrain the search for new physics. In contrast, option (2) arguably supports the philosophy that the limits of mathematics are dependent on physical laws. This viewpoint does not encourage the hope that computer scientific insights may constrain physics, but instead that new physics may extend the limits of algorithms and mathematics.

An important objection to this argument is the following: that, despite its origin in physics, BQP is a fully mathemat- ical notion, since the underlying concepts, namely the superposition principle and the tensor product structure of Hilbert space, are fully mathematical; and that, a classical computer scientist, accustomed to a different mathematical framework (namely that of discrete mathematics with composite systems described in terms of the cartesian product) may have found quantum computation a little unfamiliar, simply because of unfamiliar mathematics, rather than because they involve physics in any essential way.

This point merits further consideration, but we briefly note the following: that, unless one adopts option (3), one probably has no way, except by empirical observation of the physical world, to fix what one would regard as the most powerful possible "reasonable" model of computation, both in the sense of computational complexity and of computability. For example, to one who adopts the viewpoint of option (1) or (2), there would be no fundamental explanation for his claim that quantum computers can exist in nature, but machines such as (say) nondeterministic computers cannot.

It remains to concretize option (3) in the form of a model of measurement that accounts for a bounded degree of superposition. Such a model had been proposed by us earlier [36, 37], which we briefly review in the next Section.

\section{COMPUTATIONAL MODEL FOR QUANTUM MEASUREMENT}

This section briefly discusses a model of quantum measurement, called the computational model for quantum measurement (CMQM), which is a particular realization of option (3). A fundamental assumption of the CMQM is that Hilbert space is discrete [36, 37]. The idea of a discrete Hilbert space has been independently arrived at in Ref. [44] on quantum gravity grounds. Here discretization means that the description of any quantum state with respect to some reference basis in a finite dimensional Hilbert space requires only a finite number $(\mu)$ of bits. We denote by $\mathcal{H}_{\mu}$ the Hilbert space $\mathcal{H}$ discretized at $\mu$-bit accuracy. Parameter $\mu$ specifies an intrinsic limit on the resolution of states, and not an effective limit due to practical constraints. In a more detailed model, $\mu$ need not be fixed, but only upper-bounded, and discretization may not mean a lattice structure.

A state $|\psi\rangle \in \mathcal{H}_{\mu}$ is described by $\mu$ bits per amplitude $(\mu / 2$ for the real and imaginary parts). Thus the full state is specified by $D \mu$ bits, where $D$ is the Hilbert space dimension (Actually, $(D-1) \mu$ bits suffice because of normalization. However, for simplicity, we will ignore this detail.) The sub-physical computational rate corresponding to a system's evolution when driven by a Hamiltonian $E_{j}|j\rangle\langle j|$ is $\mathcal{F}\left(D, E_{j}\right)=\frac{2^{\mu / 2}}{\hbar} \sum_{j} E_{j} \equiv \frac{2^{\mu / 2} D \bar{E}}{\hbar}$ operations per second (ops) [36, 37]. Unitarity and normalization hold true only to $\mu$-bit precision. In principle, continuous $S U(N)$ group structure can be obtained in the long wavelength limit from discrete symmetry. Thus, the discretization is not necessarily inconsistent with the observed apparent continuous evolution of quantum systems [4].

A consequence of finite $\mu$ is that the degree of superposition of a coherently evolving system is bounded above by $D_{\max }=2^{\mu}$ since in a larger Hilbert space, not all amplitudes can be resolved. Therefore, the coherent evolution of any physical system can proceed along at most a finite number, $2^{\mu}$, of parallel superpositional pathways (terms in a coherent 
superposition). The number of quantum TMs or programs in CMQM is thus only countably infinite, and we recover uncomputability. In an arbitrary dynamical situation, a Hilbert space of dimension larger that $2^{\mu}$ may become energetically available to the system. If in this situation the 'loss of probability' through unresolvability of amplitudes is sufficiently small, then the loss is deemed insignificant. Eg., given $\alpha$, for sufficiently large $\mu$, a coherent state $|\alpha\rangle$ and its finite equivalent $\left|\alpha_{\mu}\right\rangle$ in $\mathcal{H}_{\mu}$ will be hardly distinguishable in practice [37].

On the other hand, if via interactions large entanglement is generated, then the unresolvability of the state, and the resultant loss of amplitude information, are arguably no longer insignificant. In Ref. [37], we introduced a simple entanglement monotone as a suitable measure of entanglement resolvable at $\mu$ bits ( $\mu$-bit resolvable or $\mu$-resolvable). Given a set $S$ of particles, let $S\left(\rho_{j}\right)$ denote the single particle marginal entropy to $\mu$-bit precision and $\mathcal{T}$ the set of all non-vanishing proper subsets of $S$. We define $\mu$-resolvable entanglement by:

$$
\xi_{\mu}^{(N)}= \begin{cases}\sum_{j=1}^{N} S\left(\rho_{j}\right) & \text { if }\left(\lambda_{+}\right)_{y} \geq 2^{-\mu / 2} \forall y \in \mathcal{T} . \\ 0 & \text { otherwise, }\end{cases}
$$

where $\left(\lambda_{+}\right)_{y}$ is 2nd largest eigenvalue (at $\mu$-bit precision) of $\operatorname{tr}_{y}(|\Psi\rangle\langle\Psi|)=\operatorname{tr}_{\bar{y}}(|\Psi\rangle\langle\Psi|)$. The idea is that two systems are not resolvably entangled if the Schmidt representation of their joint state contains only one coefficient resolvable at $\mu$-bit precision. An $N$-partite system possesses genuine $N$-partite $\mu$-resolvable entanglement only if every bipartite division reveals $\mu$-resolvable entanglement. Two systems that are not $\mu$-resolvably entangled are said to be $\mu$-separable.

Consider a system of $N$ particles with $D \ll 2^{\mu}$, but $D^{N}>2^{\mu}$. When separable, the system's state is resolvable. But in a regime of high interaction, $\xi_{\mu}^{(N)} \approx N \log D>\mu$, so that the loss of amplitude information can be significant. Significant unresolvability leads to computational instability: that is, the sub-physical simulation of the physical system at $\mu$-bit precision becomes very noisy. According to CMQM, 'collapse of the wavefunction' is an error-preventive response to computational instability, whereby the system is abruptly re-set from massive entanglement $(\xi \approx \mu)$ to a computationally stable state (with $\xi \ll \mu$ ), which may or may not be a product state in terms of the most fundamental degrees of freedom of the system.

Wavefunction collapse is thus understood as an algorithmic (rather than dynamic) process or transition. It can be shown that repeated cycles of collapse and episodes of $\mu$-unitary evolution lead to macro-classicality compatible with the decoherence of an open system [36, 37]. An implication for quantum computation is that asymptotically, the power of QCs is not BQP but BPP, since the degree of superposition (the degree of quantum parallelism) is upper-bounded by $2^{\mu}$. A quantum computer of more than $\mu$ strongly interacting qubits will tend to collapse rapidly, hardly manifesting non-classical behaviour. Ref. [44] obtain a similar result starting from the assumption of discreteness of space.

\section{RELATIVITY AND QUANTUM GRAVITY}

Earlier we noted that the no-signaling feature reduces to the assumption of an algorithmic universe built on efficient algorithms. But we observe that no-signaling only implies localism in the sense that any signal should be mediated by material motion. It does not imply that there is an upperbound (namely, c) to the speed of material motion, which Relativity does. It is not clear that these two versions of localism may be related but we conjecture they are.

Since the cardinality of space or time taken as a continuum is the same as that of the set of functions $f: \mathbf{N} \mapsto\{0,1\}$, the possibility seems to arise of analog computers with superTuring power, at least in a noiseless classical world. One can imagine solving the halting problem in this rather exotic fashion: by executing the first step of computation in half a second, the second in the next $1 / 4$ second, the third in $1 / 8$ of a second in the subsequent interval, and so on until at the end of 1 second, the halting problem has been solved! Also, given the ability to compute $x+y, x-y, x y, x / y$ and $\lfloor x\rfloor$ in one step, where $x$ and $y$ are any unlimited-precision real numbers, NP-complete and even PSPACE-complete problems are classically solvable in polynomial time [45].

For this reason, under option (3), we would expect that physical space and time must also be disrete. In fact, reasonable grounds lead us to expect that finite $\mu$ implies discreteness of space and time [37]. Spacetime discreteness is of course an idea familiar in certain approaches to quantum gravity [46]. As the discreteness of spacetime rules out spaceor time-based analog computers, we again recover properties favoring computability and polynomiality of the universe.

\section{DISCUSSION}

The present work may be summarized as an effort to take CCAP seriously as a fundamental physical principle. That the proposition of the universe's algorithmicity can provide an economic explanation for such a wide range of basic physical laws as quantum mechanical linearity, unitarity, signallocality, the Born rule, and macroscopic classicality is justification for the belief that the search for fundamental physical laws can benefit from examining constraints coming from computation theory. Although many physicists (as against computer scientists) may be skeptical, we believe it is worth taking this idea farther and asking whether all qualitative features of physical laws can be reduced to results in the logical foundations of mathematics and computation theory.

It is a deep-rooted belief of scientists that the laws of physics should be unified into a single deeper law, simply because it seems unlikely (though not impossible) that the universe is fundamentally a patchwork of independent, basic laws. Similarly, we also expect that the mathematical structure that physical laws will assume at a sufficiently deep level may force us to resolve our ambiguity towards such profound and basic mathematical concepts as continuity and infinitessimals in real analysis, and those like infinity in the logical foundations of mathematics [47]. We believe the present approach indicates one way to address this issue, by suggesting a concrete connection between computation theory and physical law. Further, it has important implications for the philosophy of mathematics [48]. It is not unreasonable to regard logic, mathematics or classical computation theory as independent of the "accident" of physical laws and intrinsic to the "laws of thought", however one might conceive. them The discovery of Shor's celebrated algorithm, when interpretted under option (2), would seem to undermine this belief. In contrast, option (3) tends to affirm it, and gives us confidence to believe that 
NPHA and CTT should be true in any instance of the universe. It can thus help constrain the search for new physical laws on the road to fundamental theories such as a theory of quantum gravity or string theory.

Are there tests of the the model of the algorithmic universe? More simply, is it falsifiable? Clearly, any unequivocal proof that wavefunction collapse does not happen will falsify it. But this may be difficult to test, given that CMQM is hardly distinghishable from the effect of decoherence nonselectively, and experimental tests of decoherence performed thus far are incapable of differentiating the effect of decoherence from that of decoherence terminated by a collapse [37]. This is an important issue we hope to address in the future.

I thank Dr. Piyush Kurur, Prof. J. Gruska, Mr. Sudhir K. Singh for comments and discussions. I am thankful to the anonymous referees for constructive suggestions.
[1] M. Nielsen and I. Chuang, Quantum Computation and Quantum Information, Cambridge:Cambridge Univ. Press, 2002.

[2] J. Gruska. Quantum computing, London:McGraw-Hill, 1999.

[3] H. Halvorson, eprint quant-ph/0310101.

[4] H. Hardy, eprint quant-ph/0111068; quant-ph/0101012

[5] S. Lloyd, Phys. Rev. Lett. 88, 237901 (2002).

[6] J. Bekenstein, Contemp. Phys. 45, 31-43 (2004).

[7] C. Sivaram, Current Science 79, 413-420 (2000).

[8] J. Ng, Phys. Rev. Lett. 86 2946-2949 (2001); ibid. 88, 139902 (2002).

[9] P. Shor, SIAM Journal of Computing 26, 1484-1509 (1997).

[10] R. T. Cahill and C. M. Klinger, Phys. Lett. A223, 313319 (1996); R. T. Cahill and C. M. Klinger, Gen. Rel. \& Grav. 32, 529-540 (2000); R. T. Cahill, C. M. Klinger and K. Kitto, The Physicist, 37, 191-195 (2000).

[11] A. Zeilinger, Found. of Phys. 29, 631-643 (1999).

[12] S. Wolfram, A New Kind of Science, Wolfram Media, 2002.

[13] E. Fredkin, www.digitalphilosophy.org.

[14] J. A. Wheeler, At Home in the Universe, New York:American Institute of Physics, 1994.

[15] S. Aaronson, in Proc. on the Foundations of Probability and Physics Växjö, Sweden-2004, edited by A. Khrennikov; eprint quant-ph/0401062

[16] S. Aaronson, ACM SIGACT News, March 2005; eprint quant-ph/0502072

[17] A. Turing, Proc. Lond. Math. Soc. (ser 2.) 42, 230-265 (1936); 45, 161-228 (1937).

[18] D. Deutsch, Proc. of the Royal Society of London, A400, 97-111 (1985).

[19] E. Bernstein and U. Vazirani, Quantum complexity theory, Proc. the 25th Annual ACM Symposium on Theory of Computing, 11-20 (1993).

[20] C. Papadimitriou, Computational Complexity, New York:Addison-Wesley, 1994; accessible online references are www.complexityzoo.com/ and en.wikipedia.org/.

[21] A decision problem $C$ is $\mathbf{N P}$-complete if it is in NP and if every problem in NP is polynomial-time many-one reducible to it. This means that for every NP problem $L$, there exists a polynomial time algorithm which can transform any instance of $L$ into an instance of $C$ with the same truth value. As a consequence, if we had a polynomial time algorithm for $C$, we could solve all NP problems in polynomial time [20].

[22] $\mathbf{P P}$ is the class of decision problems solvable by a probabilistic $T M$ with error probability less than $1 / 2$. That is, to any (decision) problem in $\mathbf{P P}$, there is an algorithm that is allowed to make random decision based on a coin flip, such that it returns yes with with probability greater than $1 / 2$ if the answer is yes, and returns yes with prob- ability at most $1 / 2$ if the answer is no. It can be shown that $\mathbf{N P} \subseteq \mathbf{P P}$. Strict containment is believed but not proven. Note that unlike in the case of BPP, acceptance by a 'clear' majority is not required. The error probability can be exponentially close to $1 / 2$. This gives extra power to $\mathbf{P P}$ to capture the classes such as NP and co-NP.

[23] D. S Abrams and S. Lloyd, Phys. Rev. Lett. 81, 39923995 (1998).

[24] A. Einstein, B. Podolsky and N. Rosen, Phys. Rev. 47, 777-780 (1935).

[25] N. Gisin, Phys. Lett. A, 143, 1-2 (1990).

[26] J. Polchinski, Phys. Rev. Lett. 66, 397-400 (1991).

[27] M. Znidaric and M. Horvat, Phys.Rev.A. 73, 022329 (2006); eprint quant-ph/0509162

[28] G. Chaitin, eprint math.HO/0411418

[29] K. Gödel, Monatschrifte für Mathematik und Physik 38, 173-198 (1931).

[30] M. A. Nielsen, Phys. Rev. Lett. 79 2915-2918, (1997).

[31] C. H. Bennett, Ethan Bernstein, Gilles Brassard and U. Vazirani, eprint quant-ph/9701001

[32] J. E. Hopcroft and J. D. Ullman, Introduction to Automata Theory, Langauges and Computation WileyEastern, 2003.

[33] M. Ziegler, quant-ph/0410141.

[34] M. Davis, Computability and Unsolvability, New York:Dover, 1982.

[35] M. Schlosshauer, Rev. Mod. Phys. 76, 1267-1305 (2004); eprint quant-ph/0312059.

[36] R. Srikanth, Quantum Information Processing 2, 153 199 (2003); eprint quant-ph/0302160.

[37] R. Srikanth, eprint quant-ph/0601062

[38] C. Kiefer and E. Joos, eprint quant-ph/9803052

[39] H. D. Zeh, Lect. Notes Phys. 538 19-42 (2000); eprint quant-ph/9905004.

[40] W. H. Zurek, Rev. Mod. Phys. 75, 715-775 (2003).

[41] S. Aaronson, quant-ph/0507242.

[42] Oded Goldreich, wisdom.weizman.ac.il/ oded/onqc.html, 2004.

[43] A. Bassi and G.-C. Ghirardi, Phys. Lett. A 275, 373-381 (2000); eprint quant-ph/0009020

[44] R. Buniy, S. Hsu, A. Zee, Phys. Lett. B 630, 68-72 (2005); eprint hep-th/0508039

[45] A. Schönhage, in Proc. Intl. Colloquium on Automata, Languages and Programming, 1979, pp. 520-529.

[46] L. Smolin, Three Roads to Quantum Gravity, Weidenfeld and Nicolson, 2000.

[47] H. Weyl The Continuum: A Critical Examination of the Foundations of Analysis, Dover, 1994.

[48] B. Russell, Introduction to Mathematical Philosophy, Routledge, 1993. 